\title{
ON THE ORIGIN \\ OF THE COSMIC ELEMENTS AND THE NUCLEAR HISTORY OF THE UNIVERSE
}

n Jordi José 1 , Martin Asplund ${ }^{2}$, Corinne Charbonel ${ }^{3}$, Isabelle Cherchneff ${ }^{4}$, Roland Diehl ${ }^{5}$, Andreas Korn ${ }^{6}$ and Friedrich-Karl Thielemann ${ }^{4}$

【 ${ }^{1}$ Technical University of Catalonia, Barcelona, Spain $-{ }^{2}$ Australian National University, Canberra, Australia $-{ }^{3}$ University of Geneva, Switzerland $-{ }^{4}$ University of Basel, Switzerland $-{ }^{5}$ Max-Planck-Institute for Extraterrestrial Physics, Garching, Germany Uppsala University, Sweden - DOI: http://dx.doi.org/10.1051/epn/2016401

The quest for the energy source of stars, capable of maintaining their long-lasting brightness, has puzzled physicists during centuries. Early suggestions, due to Julius R. von Mayer, John James Waterson, Hermann von Helmholtz, and William Thomson (Lord Kelvin), among others, relied on the conversion of gravitational potential energy into heat. However, the age of the Sun inferred in this framework was only a few million years, a value clearly at odds with estimates based on geological records. 


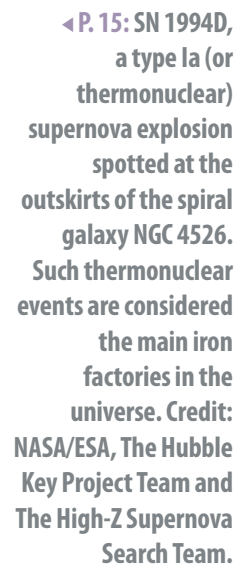

a type la (or

thermonuclear) supernova explosion

spotted at the outskirts of the spiral

galaxy NGC 4526.

Such thermonuclear

events are considered

the main iron

factories in the

universe. Credit:

NASA/ESA, The Hubble

Key Project Team and The High-Z Supernova

Search Team.

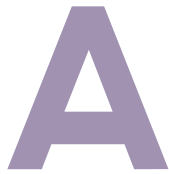

$\mathrm{fter}$ the serendipitous discovery of radioactivity by Antoine H. Becquerel in 1896, the focus shifted toward nuclear energy. Following a series of atomic mass measurements, Francis W. Aston concluded in 1920 that four individual hydrogen nuclei are heavier than a helium nucleus [1] This result led Arthur S. Eddington [2] to suggest that the energy source of the Sun lies in the conversion of hydrogen into helium, thus releasing this extra 'binding energy' deep inside the star. A major step forward in understanding the fusion of atomic nuclei was provided by experiments on Coulomb barrier penetration, performed by George Gamow [3] and Ronald W. Gurney and Edward U. Condon [4], which led Robert Atkinson and Fritz Houtermans [5] to conclude that quantum tunneling plays a key role in the energy generation in stars through nuclear fusion.

The earliest stage in the nuclear history of a star is (central) hydrogen fusion with helium as final product. Soon, two different pathways for hydrogen fusion were identified: the so-called proton-proton chains [6,7] and the CNO cycle $[8,9]$. This pioneering work paved the road for the first self-consistent studies of element production in stars, the so-called nucleosynthesis theory, by Fred Hoyle $[10,11]$. Equally influential was the compilation of Solar System abundances by Hans Suess and Harold Urey [12]: Plotted as a function of mass number, $\mathrm{A}$, the distribution

v FIG. 1: Composite image of a historical supernova remnant (SN 1006), likely a type la supernova, located at 7100 light-years from Earth. Type la supernovae are prominent Fe factories in the universe. Credit: X-ray: NASA/CXC/Rutgers/G. Cassam-Chenai, J. Hughes et al.; Radio: NRAO/AUI/NSF/GBT/VLA/ Dyer, Maddalena, and Cornwell; Optical: Middlebury College/F. Winkler, NOAO/AURA/NSF/CTIO Schmidt and DSS. Source: Wikipedia.

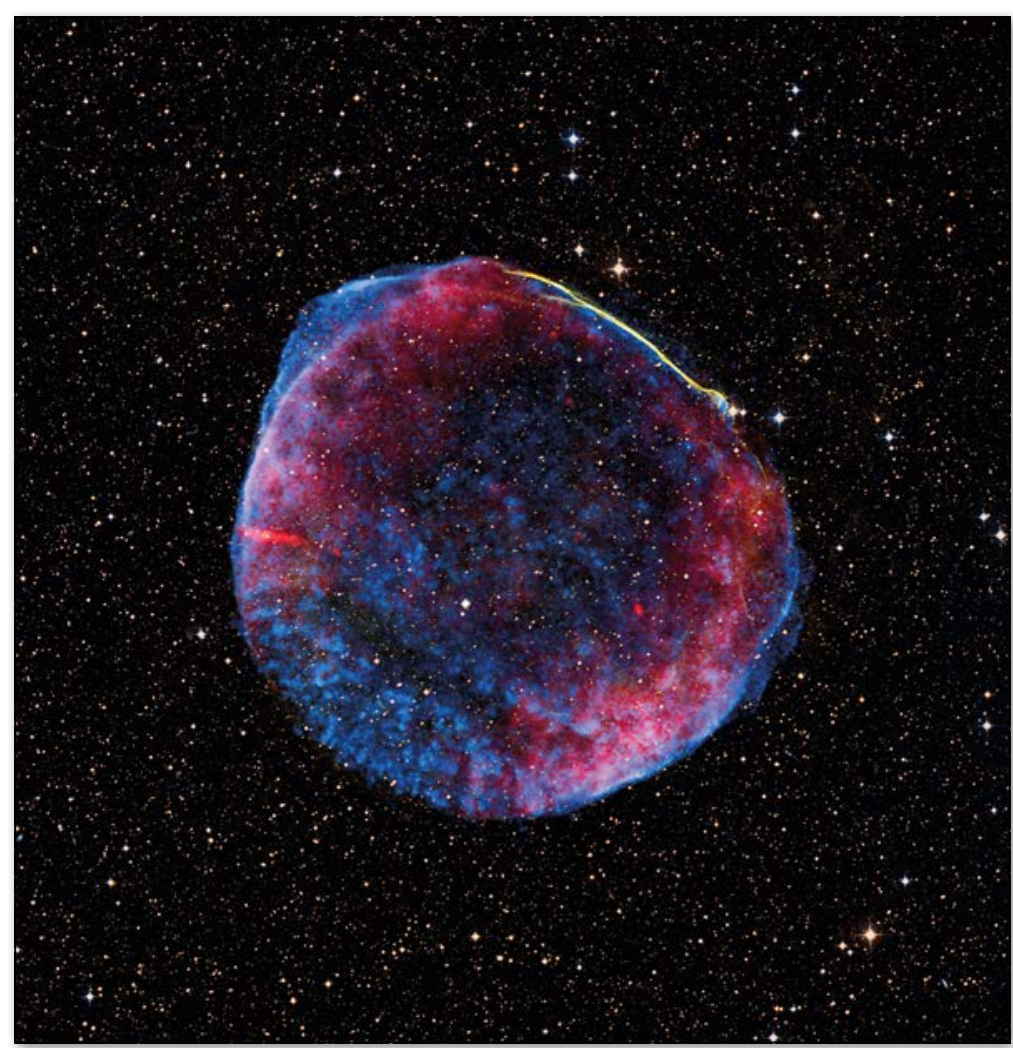

of abundances shows a complex pattern across ten orders of magnitude of abundances, with hydrogen and helium being by far the most abundant species, with a secondary peak towards iron at $\sim 1 / 10000$ of their abundance, and again much lower abundances for heavier elements beyond. The presence of several maxima were soon attributed to a number of nuclear physics effects, such as the existence of tightly bound nuclei (e.g., $\left.{ }^{56} \mathrm{Fe}\right)$, or the role played by closed-shell configurations with magic numbers at 50, 82, and 126 nucleons.

Stars appeared as the likely sites where most of the cosmic elements were actually being cooked, but observational evidence was as yet missing. In this regard, the detection of technetium in the spectra of several giant stars by Paul W. Merrill, in 1952, provided smoking-gun evidence to this conjecture [13]. Technetium is, in fact, the lightest element with no stable isotopes. Since its longest-lived form has a rather short half-life, $\mathrm{t}_{1 / 2}\left[{ }^{98} \mathrm{Tc}\right] \sim 4.2 \mathrm{Myr}$, it was likely produced in situ, in the observed stars. Compared with the age of the Galaxy of more than 10 Gyrs, this proves that nucleosynthesis is still ongoing in the Universe. Two seminal papers, that provided the theoretical framework for the origin of the chemical species, were published shortly after, in 1957 , almost exactly a century since Darwin's treatise on the origin of biological species: the first, by Margaret Burbidge, Geoffrey Burbidge, William A. Fowler and Fred Hoyle, in Reviews of Modern Physics [14], and the second, a compilation of lecture notes known as the Chalk River report CRL-41, by Al G.W. Cameron [15].

More than half a century later, nuclear astrophysics has flourished as a truly multidisciplinary field, aimed at understanding energy production in stars and the origin of the chemical elements in the Universe (Figure 1). New tools and developments, at the crossroads of theoretical and computational astrophysics, observational astronomy, astro- and cosmochemistry, and nuclear physics, have revolutionized our understanding of the nuclear history of the universe. The use of space-borne observatories, for instance, has opened new windows, so that we can study the cosmos through multifrequency observations. Indeed, since the last decades, UV, Xand $\gamma$-ray satellites have been used simultaneously to ground-based optical and radiotelescopes to analyze the behaviour of stars and their explosions at different wavelengths. In parallel to the elemental stellar abundances inferred spectroscopically, cosmochemists are now providing isotopic abundance ratios from micron-sized presolar grains extracted from meteorites. Encapsulated in those grains, there is pristine information about the suite of nuclear processes that took place near their condensation sites in stellar outskirts, which translate into huge isotopic anomalies with respect to bulk solar-system material. The dawn of supercomputing has also provided astrophysicists with the appropriate tools 
to study the complex physical phenomena of nucleosynthesis that require a truly multidimensional approach (e.g., convective and radiative energy transport by photons and neutrinos, mixing of matter through flows by convection or stimulated by stellar rotation, and flame propagation in stars). Also, nuclear physicists have developed new experimental (and theoretical) techniques to determine nuclear interactions at or close to stellar energies, at the so-called Gamow window, thus reducing the problems associated with extrapolation of measurements from the higher energies that are accessible in the laboratory down to stellar energies. Moreover, they have also explored the properties of nuclei far from stability and of matter at and beyond nuclear densities.

\section{The EuroGENESIS Program: One Step Forward}

Understanding of the progress achieved in those various sub-fields of nuclear astrophysics and assessment of its current challenges require the combination of efforts in observational astronomy, cosmochemistry, computational astrophysics, and experimental and theoretical nuclear physics. In this framework, the EuroGENESIS Program (ESF, 2010-2013) successfully assembled about 200 specialists in these fields (from 30 research institutions and universities from 15 European countries, plus the US and Canada), into a coordinated, interdisciplinary effort aimed at understanding how matter evolved in the Universe, from the ashes of the Big Bang to its current form.

With an overall Budget estimated in 2.5 M€, EuroGENESIS was thematically arranged around four, intertwined research topics:

\section{Nucleosynthetic fingerprints of the first stars} The oldest stars of the Milky Way represent a unique window to the early Universe. Searches for ever more metal-poor stars have been conducted since the 1950s (more metal-poor meaning less chemical enrichment, meaning closer to the metal-free Big Bang) and since the 2000s we know stars with 10 million times less iron than the Sun. The chemical fingerprints in the spectra of these stars trace the nucleosynthesis and physics of the very first supernovae which likely exploded a few hundred million years after the Big Bang (corresponding to a mere $1-2 \%$ of the present age of the Universe). Within this collaboration, a diverse team of experts looked at the whole chain of steps needed to understand early Galactic chemical evolution: from finding the most metal-poor and oldest stars and refining the analysis using state-ofthe-art simulations of the light-emitting regions of these stars (in terms of hydrodynamic and diffusive processes), to properly interpreting the chemical make-up of these objects and of the galaxy and its substructures (in particular halo and globular clusters) by means of models of rotating stars.

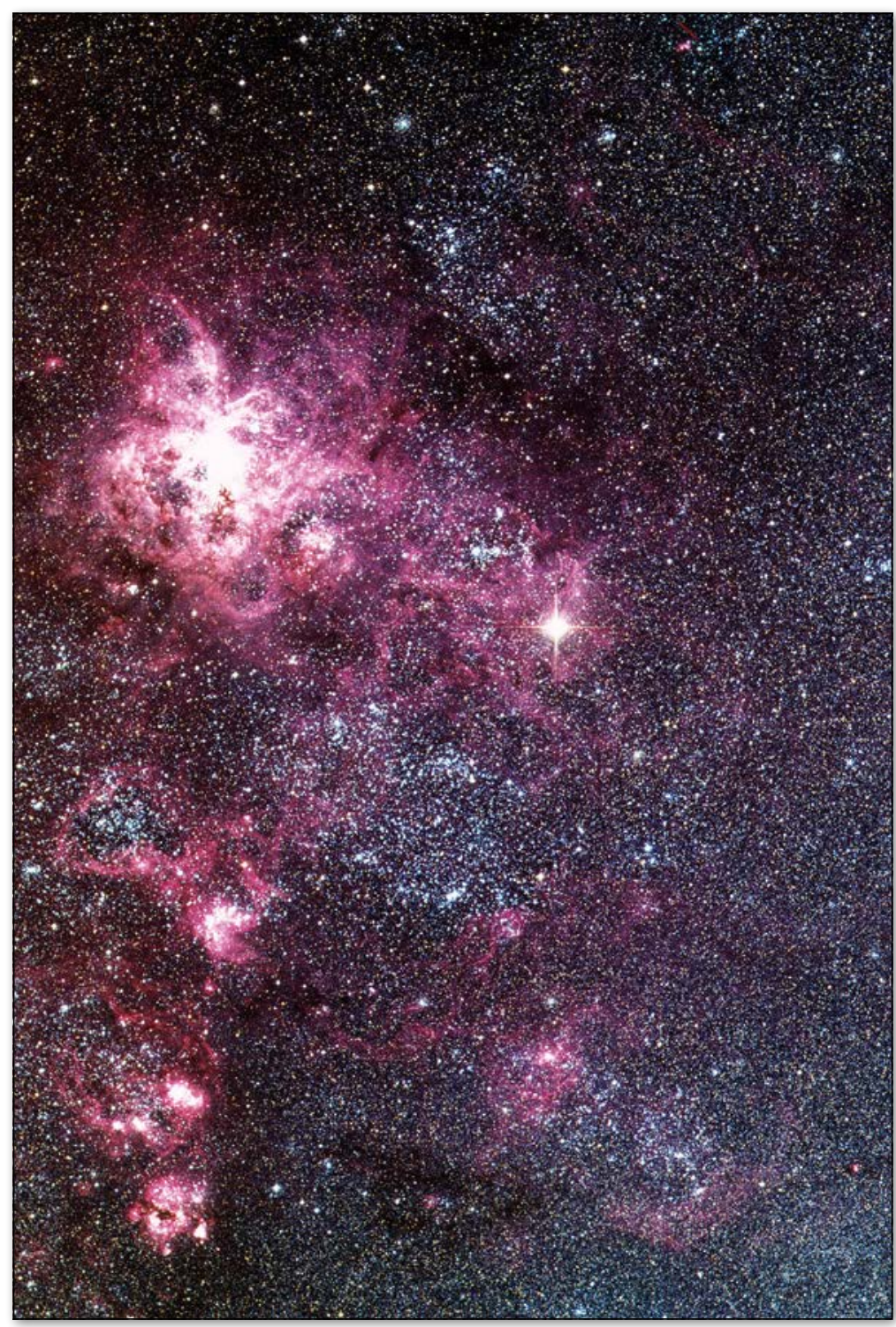

$\triangle$ FIG. 2: SN 1987A, the closest supernova detected since the invention of the telescope. The object corresponds to the very bright, massive star in the middle right of the Tarantula Nebula, in the Large Magellanic Cloud. At the time of this picture, SN 1987A was visible with the naked eye. Credit: ESO. Source: http://www.eso.org/public/images/es00708a/, released into public domain by ESO.

Among the most relevant results achieved by the different researchers of this collaboration, one could mention: - Discovery of the most iron-deficient halo star known using the SkyMapper telescope;

Discovery of extremely metal-poor stars in the Milky Way bulge, predicted to be the oldest stars known in the Universe;

Work on metal-poor globular clusters showing that the surface compositions of little-evolved stars are systematically affected by atomic diffusion, lowering individual chemical abundances by up to $60 \%$;

Improved modelling of the ${ }^{6} \mathrm{Li} /{ }^{7} \mathrm{Li}$ ratio in metal-poor stars;

A 300-night spectroscopic survey of the Milky Way at the Very Large Telescope;

Study of the effects of internal gravity waves, rotation-induced mixing, and atomic diffusion on the evolution and chemical properties of metal-poor low-mass stars; 


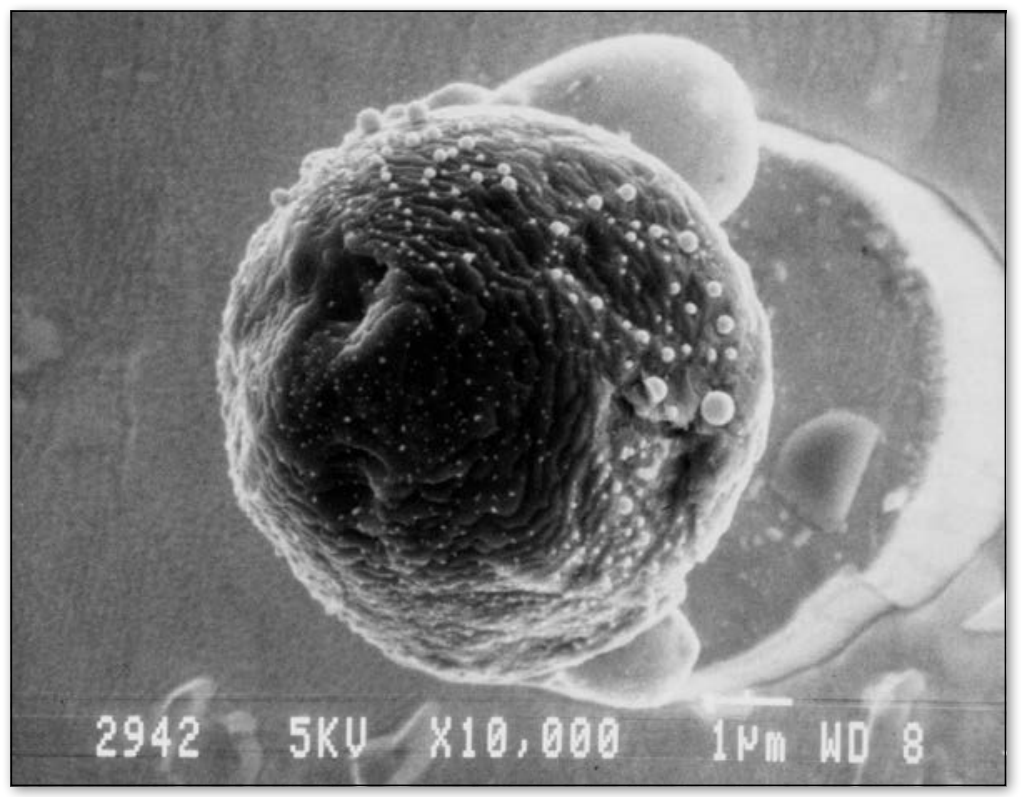

$\triangle \mathrm{FIG}$.3: A number of nova and supernova candidate grains have been identified through Secondary lon Mass Spectrometry. Image of the graphite grain $\mathrm{KFC1a-511,} \mathrm{courtesy} \mathrm{of} \mathrm{Sachiko} \mathrm{Amari.}$

- Study of the nucleosynthetic signatures of the first rotating massive stars on the long-lived low-mass stars in globular clusters;

- Improved spectroscopic analysis techniques for metal-poor stars to determine their chemical compositions.

\section{Massive stars as agents of chemical evolution} This collaboration consisted of groups with expertise in experimental and theoretical nuclear physics, stellar evolution and explosion (e.g., supernovae; see Figure 2), resulting nucleosynthesis predictions, and their impact on the local environment, as well as the overall abundance evolution of galaxies. Observers from various astronomy windows as well as meteoritic studies complemented this collaboration. This led to a variety of inter-disciplinary work: Detailed comparisons of different stellar evolution codes, in order to test the treatment of mixing and other ingredients with the aim to measure the obtained convergence in our understanding of stellar evolution. Comparison of predictions with observations resulted in incentives to perform nuclear experiments (partially underground). Theoretical predictions for reactions in early and late phases of stellar evolution and explosions were tested with respect to their impact. Supernova core collapse simulations probed the nuclear equations of state and predictions for nucleosynthesis ejecta. Finally, employing these results in chemical evolution processes of the Galaxy and its building blocks, permitted to utilize a variety of astronomical constraints as a feedback for individual stellar modelling as well as the treatment of galactic evolution. The result was the formation of a community with many new and direct interactions, connecting the knowledge from nuclear reactions over stars and supernova simulations to observations of the diffuse interstellar gas and individual objects as well as their imprint in galactic evolution.

Among the large number of achievements reached in this Collaboration, a few are worth emphasising:

- A highly improved nuclear input basis has been set, from low-energy fusion reactions, n-captures, charged-particle reactions for explosive conditions (from experiment and theory), nuclear masses far from stability, fission barriers and fission fragment distributions, electron-captures and neutrino induced reactions; Improved understanding was obtained of uncertainties in stellar evolution, comparing different evolution codes, strong progress in modelling stellar evolution with rotation, including the effect on hydrostatic nucleosynthesis ejecta and wind composition;

- Major progress in supernova modelling and successful explosions emerged, and now we are close to being able to perform nucleosynthesis calculations in multi-D models, which - together with other observational features - can test these models and the utilized physics ingredients;

Intermediate approaches in 1D, with the aim to mimic multi-D modelling, permitted to perform large-scale and extensive nucleosynthesis predictions which can test the effect of weak interactions on the abundance features of the innermost ejected zones;

- Understanding the r-process site (i.e., the stellar factories of half of the neutron-rich nuclei heavier than iron, created through rapid neutron captures) remains a challenge, but our understanding was strongly advanced, addressing in great detail neutrino winds, jets from fast rotating, highly magnetized core collapse supernovae, and neutron star mergers, and performing also tests of these sites with their impact on galactic chemical evolution.

\section{Cosmic dust grains as diagnostic for massive stars}

This was a multidisciplinary collaboration, whose objectives were to advance knowledge of the formation of cosmic dust in supernovae, the reprocessing of dust in supernova remnants, and the chemical seeding with isotopes and dust of the interstellar medium and the solar nebula by supernovae. The collaboration uniquely gathered several research groups from four different fields of astrophysics (astrochemistry, astronomy, experimental physics of meteorites and nuclear physics) and delivered several important outcomes on the production of dust by core-collapse supernovae. The collaboration successfully delivered the following results:

Core-collapse supernovae are significant producers of several dust components (silicates, alumina, carbon, silicon carbide, pure metals and iron grains) locally and in the early universe. The formation of dust goes along with the synthesis of simple molecules in the ejected material, such as $\mathrm{CO}, \mathrm{SiO}, \mathrm{SO}$ and $\mathrm{SiS}$. The recent detection of molecules with the Atacama Large Millimetre Array, 
ALMA, in the young supernova remnant SN1987A has confirmed these predictions. The production of dust occurs in various zones of the ejected material a few months after the supernova explosion and the dust mass gradually increases over a period of five years from small values ( $10^{-4}$ solar masses) to large values ( 0.1 solar masses). This gradual growth provides a possible explanation for the discrepancy between the small amounts of dust formed at early post-explosion times and the high dust masses derived from recent observations of supernova remnants like SN1987A, Cassiopeia A and the Crab nebula;

Sputtering of dust by shocks in the remnant phase is highly dependent on the clumpiness of the gas in the remnant. Large dust grains (>0.1 microns; see Figure 3 ) survive shocks and can be injected in the interstellar medium. These grains will finish their life in proto-stellar nebula and be incorporated in meteorites in the solar nebula. Observation time on the space telescope Herschel was obtained to study dense gas clumps rich in molecules and dust in the supernova remnant Cassiopeia A. These observations confirmed the existence of molecules formed in the supernova that are later shocked in the remnant, with the detection of warm $\mathrm{CO}$ emission lines;

A sample of a deep-sea manganese crust that showed the ${ }^{60} \mathrm{Fe}$ "supernova- signal" was searched for live ${ }^{244} \mathrm{Pu}$, and revealed unexpected low fluxes. This finding indicates that no significant actinide nucleosynthesis happened within the last few hundred million years. This is incompatible with normal actinide production in supernovae and suggests that a rare event rate, i.e., a small subset of supernovae or neutron-star mergers, has seeded the solar nebula.

\section{Physics of compact objects:} explosive nucleosynthesis and evolution

Many stars form binary or multiple systems, with a fraction hosting one or two degenerate objects (white dwarfs and/or neutron stars) in short-period orbits, such that mass transfer episodes onto the degenerate component ensue. This scenario is the framework for a suite of violent stellar events, such as type Ia supernovae, classical novae, $\mathrm{X}$-ray bursts, or stellar mergers (involving white dwarfs, neutron stars and black holes). The expected nucleosynthesis accompanying these cataclysmic events is very rich: classical novae are driven by proton-capture reactions in competition with $\beta$-decays, proceeding close to the valley of stability, up to Ca. Type I X-ray bursts are powered by a suite of nuclear processes, including the rp-process (rapid p-captures and $\beta$-decays), the $3 \alpha$-reaction, and the ap-process (a sequence of ( $\alpha, p)$ and $(p, \gamma)$ reactions); here, the nuclear flow proceeds far away from the valley of stability, merging with the proton drip-line beyond $\mathrm{A}=38$, and reaching eventually the SnSbTe-mass region, or beyond. In type Ia supernovae, the detailed abundances of the freshly synthesized elements depend on the peak temperature

\section{The experience achieved in the multi-national, multi-institute EuroGENESIS project has built and organized a European Community in the wide field of nuclear astrophysics.}

reached and on the excess of neutrons and protons (which depend in turn on the metallicity of the white dwarf progenitor as well as on the density at which the thermonuclear runaway occurs); they constitute the major factory of Fe-peak elements in the Galaxy, and roughly speaking, the abundance pattern of their ejecta is the result of four different burning regimes: nuclear statistical equilibrium (NSE) and incomplete $\mathrm{Si}-, \mathrm{O}-$, and C-Ne-burning. A suite of different nuclear processes are expected to occur during stellar mergers (indeed, neutron star mergers have been suggested as a possible site for the r-process).

This collaboration consisted of groups with expertise in experimental and theoretical nuclear physics, computational hydrodynamics (with emphasis in multidimensional simulations of stellar explosions and on their associated high-energy emission in X-and gamma-rays), and cosmochemistry (through laboratory analysis of presolar grains), from 11 institutions.

Among the most relevant results achieved in this area by the different components of the Collaboration, one could mention:

- 3-D simulations of nucleosynthesis accompanying double white dwarf mergers, with emphasis on

VFIG.4:Two-dimensional snapshots of the development of hydrodynamic instabilities, in a 3-D simulation of mixing at the core-envelope interface during a nova explosion, calculated with the hydrodynamic code FLASH (adapted from Casanova et al. 2011, Nature, 478, 490).
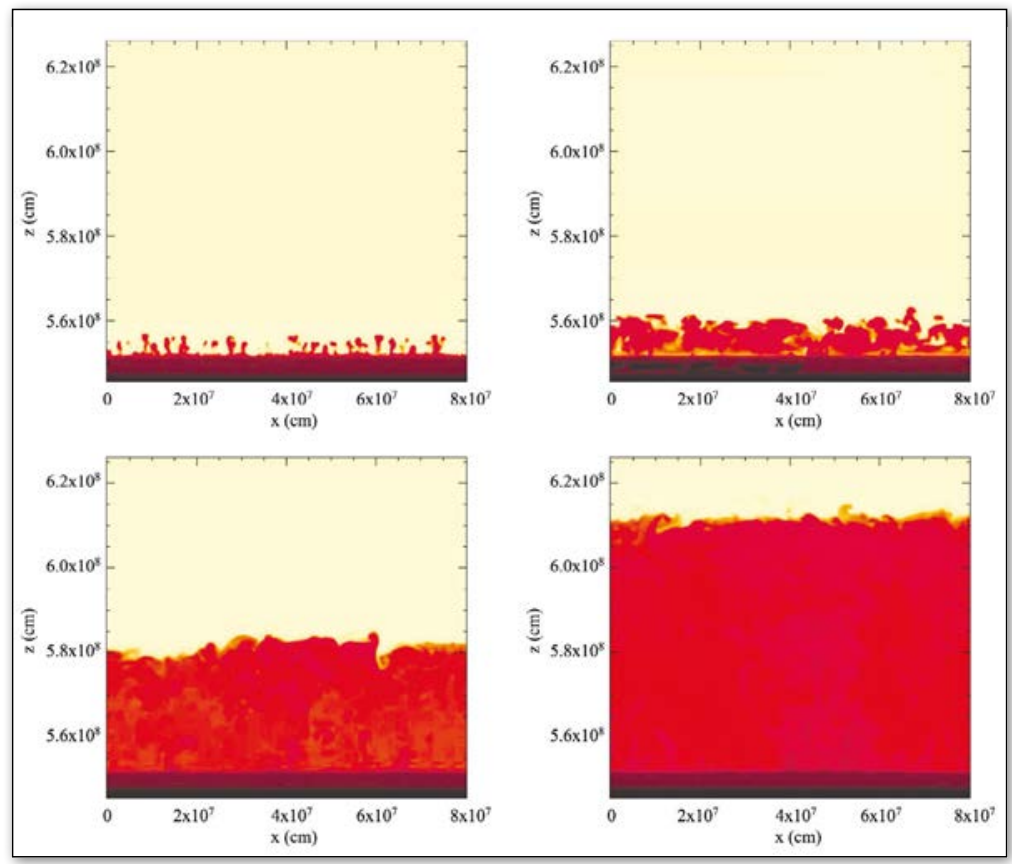


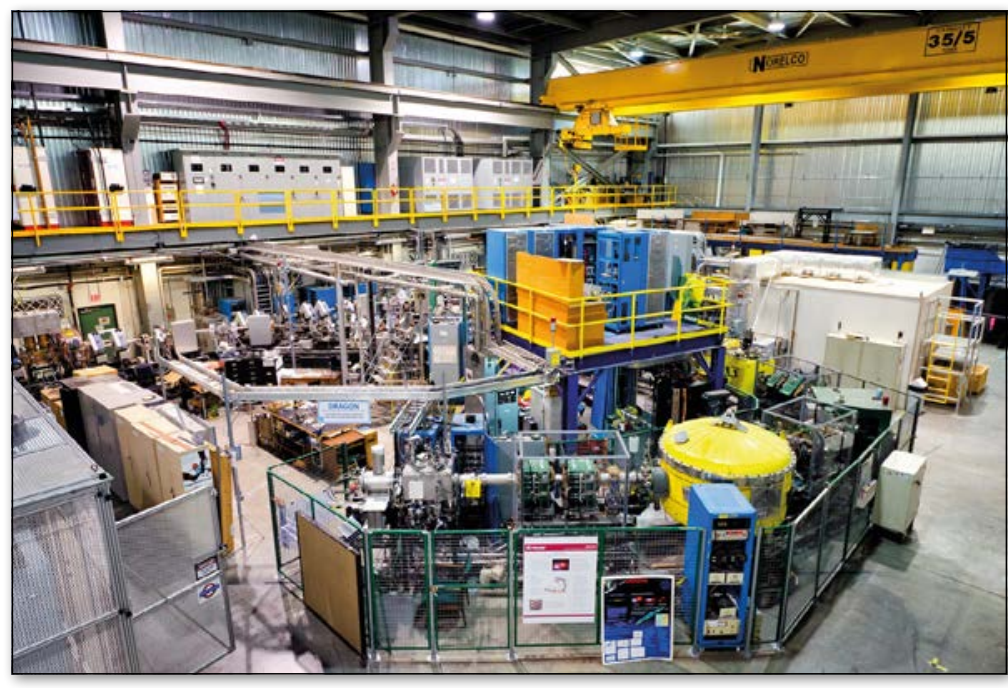

$\triangle$ FIG. 5: DRAGON an example of recoil mass separator for the study of nuclear reactions of astrophysica interest, located in the ISAC facility at TRIUMF (Vancouver). Image courtesy of Steven 0ates.

Li production and on the origin of $\mathrm{R}$ Cor Bor stars (a variety of hydrogen-deficient stars with high carbon abundances);

- New nuclear reaction-rate compilation based on Monte Carlo method. Improvements on a suite of reaction rates of interest for nova nucleosynthesis;

First 3-D simulation of mixing at the core-envelope interface during nova outbursts (Figure 4);
Identification of a number of oxide grains of a putative nova origin;

Identification of the most relevant nuclear uncertainties affecting type Ia supernova and type I X-ray burst nucleosynthesis predictions.

\section{Conclusion}

The experience achieved in the multi-national, multi-institute EuroGENESIS project has built and organized a European Community in the wide field of nuclear astrophysics. It led to new and effective interactions and information flow across the scattered and widely-spread and individually-small research groups of nuclear physics experimentalists (Figure 5), theorists, modellers and astrophysical observers. This permitted joint rather than isolated actions. It probably laid the ground work for future joint explorations at a trans-national level, necessary to make break-through advances in such a complex and inter-related field that spans from the microcosmos of nuclear forces to the macroscopic cosmic objects and the chemical evolution across the entire universe.

\section{About the authors}

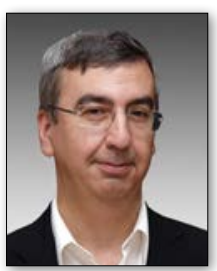

Jordi José (Technical Univ. Catalonia) works at the crossroads between computational astrophysics, nuclear physics and cosmochemistry. His research has focused in the modelling of stellar explosions, mostly in classical novae and X-ray bursts.

Martin Asplund (Australian National University) has broad research interests, ranging from the Sun and the origin of the Solar System, over stellar physics and extrasolar planets, to the history of the Milky Way and the origin of the elements across cosmic time.

Corinne Charbonnel (Univ. Geneva and CNRS) is a specialist in stellar evolution and nucleosynthesis, chemical and dynamical evolution of massive stellar clusters and galaxies, and planet habitability.

Isabelle Cherchneff (Univ. Basel) investigates the formation of cosmic dust in evolved stellar environments (AGB stars, massive stars and supernovae). She models the production of molecules, dust clusters and grains through an interdisciplinary approach that involves hydrodynamics, chemistry, nanoscience and astronomy.

Roland Diehl (Max-Planck-Institute for Extraterrestrial Physics and TU München) is a gamma-ray astronomer working with space telescopes for cosmic nuclear line spectroscopy. His main research interests are massive stars and supernova explosions, and their impact on the surroundings.

Andreas Korn (Uppsala Univ.) uses stellar spectroscopy to trace the chemical evolution of the cosmos. He has worked on hot, short-lived stars as well as cool, old stars in the Milky Way and its neighbors.

Friedrich-Karl Thielemann (Univ. Basel) makes use of properties of stable and unstable nuclei in order to employ them in predictions for nucleosynthesis ejecta, originating from stellar evolution and explosions (e.g., $\mathrm{X}$-ray bursts, supernovae, hypernovae, compact object mergers, gamma-ray bursts).

\section{References}

[1] F.W. Aston, Philosophical Magazine 39, 611 (1920).

[2] A.S. Eddington, Observatory 43, 341 (1920).

[3] G. Gamow, Zeitschrift für Physik 51, 204 (1928).

[4] R.W. Gurney and E.U. Condon, Physical Review 33, 127 (1929).

[5] R. d'E. Atkinson and F.G. Houtermans, Zeitschrift für Physik 54, 656 (1929).

[6] R. d'E. Atkinson, The Astrophysical Journal 84, 73 (1936).

[7] H.A. Bethe and C.L. Critchfield, Physical Review 54, 248 (1938).

[8] C.F. von Weizsäcker, Physikalische Zeitschrift 39, 633 (1938).

[9] H.A. Bethe, Physical Review 55, 434 (1939).

[10] F. Hoyle, Monthly Notices of the Royal Astronomical Society 106, 343 (1946).

[11] F. Hoyle, The Astrophysical Journal Supplement Series 1, 121 (1954).

[12] H.E. Suess and H.C. Urey, Reviews of Modern Physics 28 53 (1956).

[13] P.W. Merrill, The Astrophysical Journal 116, 21 (1952).

[14] E.M. Burbidge, G.R. Burbidge, W.A. Fowler, and F. Hoyle, Reviews of Modern Physics 29, 547 (1957).

[15] A.G.W. Cameron, Stellar Evolution, Nuclear Astrophysics, and Nucleogenesis. $2^{\text {nd }}$ Ed., Dover Publications, Mineola, New York (2013). 